\title{
Neural proliferation and restoration of neurochemical phenotypes and compromised functions following capsaicin-induced neuronal damage in the nodose ganglion of the adult rat
}

\section{Zachary Rex Gallaher ${ }^{\dagger}$, Vitaly Ryu ${ }^{\dagger}$, Rose M. Larios, Leslie K. Sprunger and Krzysztof Czaja*}

Programs in Neuroscience, Department of Veterinary Comparative Anatomy, Pharmacology, and Physiology, College of Veterinary Medicine, Washington State University, Pullman, WA, USA

Edited by:

Gerd Kempermann, Center for

Regenerative Therapies, Germany

Reviewed by:

Erno Vreugdenhil, Leiden University, Netherlands

Monika Ehrhart-Bornstein, Technical

University of Dresden, Germany

*Correspondence:

Krzysztof Czaja, Programs in

Neuroscience, Department of Veterinary Comparative Anatomy,

Pharmacology, and Physiology, College of Veterinary Medicine, Washington

State University, Pullman, WA

99164-6520, USA.

e-mail: czajak@vetmed.wsu.edu

†Zachary Rex Gallaher and Vitaly Ryu

have contributed equally to this work.
We previously reported that neuronal numbers within adult nodose ganglia (NG) were restored to normal levels 60 days following the capsaicin-induced destruction of nearly half of the neuronal population. However, the nature of this neuronal replacement is not known. Therefore, we aimed to characterize neural proliferation, neurochemical phenotypes, and functional recovery within adult rat NG neurons following capsaicin-induced damage. Sprague-Dawley rats received intraperitoneal injections of capsaicin or vehicle solution, followed by 5-bromo-2-deoxyuridine (BrdU) injections to reveal cellular proliferation. NG were collected at multiple times posttreatment (up to 300 days) and processed for immunofluorescence, RT-PCR, and dispersed cell cultures. Capsaicin-induced cellular proliferation, indicated by BrdU/Ki-67-labeled cells, suggests that lost neurons were replaced through cell division. NG cells expressed the stem cell marker, nestin, indicating that these ganglia have the capacity to generate new neurons. BrdU-incorporation within $\beta$-III tubulin-positive neuronal profiles following capsaicin suggests that proliferating cells matured to become neurons. NG neurons displayed decreased NMDAR expression up to 180-days post-capsaicin. However, both NMDAR expression within the NG and synaptophysin expression within the central target of NG neurons, the NTS, were restored to pre-injury levels by 300 days. NG cultures from capsaicin-treated rats contained bipolar neurons, normally found only during development. To test the functional recovery of NG neurons, we injected the satiety molecule, CCK. The effect of CCK on food intake was restored by 300-days post-capsaicin. This restoration may be due to the regeneration of damaged NG neurons or generation of functional neurons that replaced lost connections.

Keywords: neurogenesis, injury, BrdU, sensory,TRPV1, vagus

\section{INTRODUCTION}

Currently, definitive evidence in support of adult mammalian neurogenesis is restricted to the central nervous system (Kempermann et al., 2004; Ming and Song, 2005). The few studies that have been conducted within the peripheral nervous system (PNS) have led to contrasting conclusions. An age-related increase in the number of dorsal root ganglia (DRG) neurons has been reported in the rat (Cecchini et al., 1995; Popken and Farel, 1997; Ciaroni et al., 2000). In contrast, others have reported no such increase (Mohammed and Santer, 2001). The PNS has been reported to harbor neural precursors that proliferate in vitro and can differentiate into neurons (Namaka et al., 2001; Arora et al., 2007; Lagares et al., 2007; Liu et al., 2009). Together, the often reported age-related increase in DRG neuronal numbers and the presence of neural precursors supports the conclusion that the PNS has neurogenic potential (Geuna et al., 2002).

The neurogenic potential of the PNS is also evident following damage to adult rat vagal afferent neurons. We previously reported that neuronal losses within the nodose ganglia (NG) following capsaicin-induced neuronal death are not persistent
(Czaja et al., 2008). Systemic administration of capsaicin causes the death of TRPV1-positive primary afferent neurons in neonates (Holzer, 1991; Carobi, 1996). In adult rats, systemic capsaicin treatment produces extensive degeneration of nonmyelinated vagal afferent axons (Ritter and Dinh, 1988) and loss of spinal dorsal root C-fibers (Hiura, 2000). However, the long-term effect of systemic capsaicin treatment on the perikarya of sensory neurons in adults is not well-understood. Based on our previously reported observations (Czaja et al., 2008; Gallaher et al., 2010; Ryu et al., 2010), we hypothesized that capsaicininduced neuronal death is followed by neural proliferation and neurogenesis.

Glutamate and the NMDA glutamate receptor (NMDAR) are necessary for excitatory vagal afferent neurotransmission (Milner and Pickel, 2003; Czaja et al., 2006a,b). Additionally, the NMDAR promotes the survival of neurons formed in adulthood (Petrus et al., 2009). Therefore, we hypothesized that capsaicin-induced neuronal death and subsequent replacement would correlate with a disruption and restoration of NMDAR expression within the NG. 
In addition to restoring pre-injury phenotypes, new and regenerating neurons must incorporate into neural circuits. We previously reported that the number of peripheral vagal projections was restored following capsaicin-induced damage (Ryu et al., 2010). However, the effect of capsaicin treatment on central vagal projections is unknown. Therefore, we quantified synaptophysin-positive synapses within the primary target of NG afferents, the nucleus of the solitary tract (NTS; Jin et al., 2010). Like peripheral projections, we hypothesized that central projections will be lost following capsaicin treatment and return with time.

If NG neuronal numbers and projections are restored, lost functions may be restored as well. To test for functional restoration, we used the satiety hormone cholecystokinin (CCK; Dockray, 2009). CCK acts through vagal afferents and their targets (Peters et al., 2006). Furthermore, disrupting vagal signaling with vagotomy (Gillespie et al., 2005) or capsaicin (South and Ritter, 1988; Ritter et al., 1989) abolishes the effect of CCK. We hypothesized that the effect of CCK would return following the restoration of NG neuronal numbers and projections.

\section{MATERIALS AND METHODS ANIMALS}

Male Sprague-Dawley rats (6-weeks old, Simonsen Laboratories, Gilroy, CA, USA) were individually housed in a temperature-controlled vivarium with ad libitum access to food and water. Rats were maintained on a 12-h light-dark schedule and habituated to laboratory conditions for 7 days prior to capsaicin injections. All animal procedures were approved by the Washington State University Institutional Animal Care and Use Committee and conform to National Institutes of Health guidelines for the use of vertebrate animals (publication No. 86-23, revised 1985). All efforts were made to minimize the number of animals used and their suffering.

\section{CAPSAICIN TREATMENT}

Rats were injected intraperitoneally (i.p.) with capsaicin (total $n=50$; Sigma-Aldrich, St. Louis, MO, USA). The total capsaicin dose $(125 \mathrm{mg} / \mathrm{kg})$ was administered as a series of three injections $(25,50,50 \mathrm{mg} / \mathrm{kg})$ at an injection volume of $1 \mathrm{ml} / \mathrm{kg}$. All three injections were made within a 24 -h period $(0,6$, and $24 \mathrm{~h}$, respectively). An additional group of rats was injected with the vehicle solution (total $n=50 ; 10 \%$ ethanol in $10 \%$ Tween- 80 in $0.9 \%$ saline) at the same volumes and schedule as above. Rats were given an i.p. injection of atropine sulfate $(3 \mathrm{mg}) 5 \mathrm{~min}$ prior to the first capsaicin or vehicle injection. Rats were under general inhalation anesthesia (3.0\% isoflurane) during the capsaicin or vehicle treatment. Artificial respiration was provided as needed. The eye wipe response to mild corneal stimulation, a response mediated by the capsaicin-sensitive trigeminal innervation of the cornea, was tested to screen the effectiveness of capsaicin treatment. Briefly, a drop of $1 \%$ ammonium hydroxide was placed on the corneal surface of one eye. All control rats immediately wiped the stimulated eye. None of the capsaicin-treated rats exhibited any eye wipe response.

\section{BRDU INJECTIONS}

Rats were injected i.p. with 5-bromo-2-deoxyuridine (BrdU; 50 mg/ $\mathrm{kg}$ in $0.9 \%$ sterile $\mathrm{NaCl}$ at $10 \mathrm{mg} / \mathrm{mL}$; Sigma-Aldrich, St. Louis, MO, USA) every other day beginning $2 \mathrm{~h}$ after the last capsaicin or vehicle injection and continuing until sacrificing. On days 3 , $11,30,60,180$, and 300 after treatments, four capsaicin- and four vehicle-treated rats were anesthetized and perfused transcardially with $0.1 \mathrm{M}$ phosphate buffered saline (PBS; pH 7.4) followed by $4 \%$ paraformaldehyde in PBS.

\section{TISSUE FIXATION AND SECTIONING}

Immediately following the perfusion, NG and hindbrains were collected. NG were post-fixed in 4\% paraformaldehyde for $30 \mathrm{~min}$ and immersed overnight in a cryoprotectant solution of $18 \%$ sucrose and $0.1 \% \mathrm{NaN}_{3}$ (Sigma-Aldrich, St. Louis, MO, USA) in PBS. NG were cut longitudinally into $20-\mu \mathrm{m}$ serial sections, mounted in three series on glass slides, with every third section on the same slide. After drying on the slides, sections were rehydrated and processed for detection of selected antigens. Hindbrains were post-fixed in $4 \%$ paraformaldehyde for $2 \mathrm{~h}$ and immersed overnight in a cryoprotectant solution of $30 \%$ sucrose and $0.1 \% \mathrm{NaN}_{3}$ in PBS. Hindbrains were cut coronally in 30- $\mu \mathrm{m}$ sections throughout the rostro-caudal extent of the NTS (between bregma -12.8 and $-14.3 \mathrm{~mm}$ ) and stained for synaptophysin.

\section{IMMUNOSTAINING}

Slide-mounted sections of NG were immersed for $15 \mathrm{~min}$ in $0.1 \%$ sodium borohydride to reduce autofluorescence. Citrate buffer and hydrochloric acid were used for antigen retrieval. Sections were then incubated overnight in a blocking solution of $10 \%$ normal horse serum in Tris sodium phosphate buffer (TPBS; pH 7.4). Sections were incubated for $24 \mathrm{~h}$ at room temperature in one or a combination of the primary antisera listed and characterized in Table 1. Subsequently, sections were incubated for $2 \mathrm{~h}$ at room temperature in an appropriate mixture of secondary antisera for double staining (Alexa 488 and/or Alexa 555; 1:400; Invitrogen, Carlsbad, CA, USA). Sections were mounted in ProLong Gold Antifade Reagent with DAPI (Molecular Probes, Eugene, OR, USA) to reveal neuronal and glia nuclei. All of the primary antibodies used in this study were proven to be specific by the supplier via Western blot. Negative controls were performed by omission of primary antibodies as well as pre-incubation of the antiserum with the original peptide antigen. Both of these control procedures eliminated immunostaining.

Free-floating hindbrain sections were washed twice for $15 \mathrm{~min}$ in TPBS followed by a $30-$ min incubation in a blocking solution of $1 \%$ normal horse serum and $0.5 \%$ Triton X-100 in TPBS. Sections were then incubated for $24 \mathrm{~h}$ in polyclonal rabbit anti-synaptophysin (1:500; Synaptic Systems, Goettingen, Germany) in blocking solution. Sections were then incubated for $2 \mathrm{~h}$ in the secondary antibody

Table 1 | Primary antibodies used in the study.

\begin{tabular}{lllll}
\hline Target & Source & Cat.\# & Host & Dilution \\
\hline B-IIITubulin & Abcam & AB18207-25 & Rabbit & $1: 500$ \\
BrdU & Sigma & B8434 & Mouse & $1: 100$ \\
Ki-67 & Abcam & AB15580 & Rabbit & $1: 200$ \\
Nestin & DSHB & Rat-401s & Mouse & $1: 50$ \\
NR1 & St. Cruz Biot. & sc-1467 & Goat & $1: 100$ \\
Synaptophysin & Synaptic & 101002 & Rabbit & $1: 500$ \\
& Systems & & &
\end{tabular}


(biotinylated donkey anti-rabbit, 1:200; Jackson ImmunoResearch Laboratories, West Grove, PA, USA) in TPBS followed by a 4-h incubation in ABC complex (1:1000; Vectastain Elite Kit, Vector Laboratories, Burlingame, CA, USA). Horseradish peroxidase activity was revealed with a 5-min incubation in $0.05 \%$ DAB (SigmaAldrich) and $0.0003 \% \mathrm{H}_{2} \mathrm{O}_{2}$ in TPBS. Immunostained sections were mounted on slides, dried overnight, dehydrated through graded ethanol to xylene, and coverslipped with Permount.

\section{IMAGING AND QUANTIFICATION}

Images of the NG were viewed and captured with a Zeiss Axioplan2 or Nikon 80i imaging photomicroscope equipped with Axio Vision 4.6 or Nikon Elements AR imaging software, respectively. Corel Graphic Suite 11 was used to adjust brightness and contrast only for the purpose of making composite plates. In order to determine the total number of neurons in the NG, only $\beta$-III tubulin-positive neurons containing a DAPI-labeled nucleus were counted. Total neuron counts were performed in all of the sections from a given ganglion and corrected for split nuclei as previously described (Czaja et al., 2006a). Counts for additional antigens were performed in every third section to eliminate the likelihood of counting the same object twice.

Synaptophysin counts within hindbrain sections were performed at high magnification with a $100 \times(1.30 \mathrm{NA})$ oil objective using a Nikon 80i imaging photomicroscope equipped with Stereologer $\mathrm{CP}$-Version 2 software (Stereologer Resource Center, Chester, MD, USA). The optical fractionator was employed to obtain estimates of the total number of synaptophysin-positive synapses within the region of the NTS using the equation $N=\Sigma Q^{-}(h / t \times 1 /$ asf $\times 1 /$ ssf $)$, where $\Sigma Q$ equals the total number of objects counted for each subject, $h$ equals the height of the dissector, $t$ equals the thickness of the section, asf equals the area sampling fraction, and ssf equals the section sampling fraction (Calhoun et al., 1996).

\section{REAL-TIME PCR}

For each of the time points 3, 30, and 60 days, a minimum of three capsaicin and three vehicle rats were anesthetized (ketamine 25, xylazine $2.5 \mathrm{mg} / 100 \mathrm{~g}$ ) so the left and right NG could be collected and stored in RNALater (Cat \# AM7021, Applied Biosystems, Austin, TX, USA) at $-20^{\circ} \mathrm{C}$ until processing. RNA was isolated from pooled left and right NG using an Ambion RNAqueous kit (Cat \# AM1912, Applied Biosystems) and treated with an Ambion TURBO DNA-free kit (Cat \# AM1907, Applied Biosystems). First-strand cDNA synthesis was performed using the iScript cDNA synthesis kit (Cat \# 1708890, Bio-Rad, Hercules, CA, USA). Intron-spanning sequences of NR1 (Grin1) were designed using the Roche Universal Probe Library from rat sequences of NR1 F 5'-GCTTTTGCAGCCGTGAAC-3', and $\mathrm{R}$ 5'-GGGCTCTGCTCTACCACTCTT-3' corresponding to nucleotides 2816-2833 and 2861-2881, respectively, and run with probe \#69 (Cat \# 04688686001, Roche Applied Science, Indianapolis, IN, USA). PGP 9.5 (Uchl1) was used as a reference, $\mathrm{F}$ 5'-CCTGCTGCTGCTGTTTCC-3' and R 5'-TGTCCCTTCAGTTCCTCAATTT-3' corresponding to nucleotides 197-214 and 250-271, respectively, run with probe \#107 (Cat \# 04692268001, Roche Applied Science). RT-PCRs (20 $\mu$ L total volume) were performed in a Bio-Rad iCycler with iQ Supermix (Cat \# 170-8860, Bio-Rad). The amplification conditions were one cycle at $95^{\circ} \mathrm{C}$ for 4 min followed by 50 cycles going from $94^{\circ} \mathrm{C}$ for $20 \mathrm{~s}$ to $60^{\circ} \mathrm{C}$ for $1 \mathrm{~min}$, and completed by holding at $20^{\circ} \mathrm{C}$. Negative controls were included by substituting cDNA for ddH20. Data was analyzed using the $2^{-\Delta \Delta C t}$ method.

\section{DISSOCIATED CELL CULTURES}

For each of the time points 3, 30, and 60 days, a minimum of three capsaicin and three vehicle rats were anesthetized (ketamine 25, xylazine $2.5 \mathrm{mg} / 100 \mathrm{~g}$ ) so the NG could be collected and placed in ice-cold $\mathrm{Ca}^{2+}$ and $\mathrm{Mg}^{2+}$-free HBSS supplemented with antibiotic (penicillin-streptomycin, $100 \mathrm{U} / \mathrm{ml}$ and $100 \mathrm{~g} / \mathrm{ml}$, respectively). NG were cleaned of connective tissue and sliced into 1-mm-length segments using a sterile scalpel blade. NG neurons were dissociated in $3 \mathrm{ml}$ of digestion buffer $(1 \mathrm{mg} / \mathrm{ml}$ dispase II and $1 \mathrm{mg} / \mathrm{ml}$ collagenase type Ia in HBSS) for $90 \mathrm{~min}$ at $37^{\circ} \mathrm{C}$. Tissue residues were mechanically separated into single cells by gentle triturating of the suspension through glass pipettes until a homogenous solution was seen. Dispersed cells were then washed with HEPESbuffered DMEM (HDMEM) and plated onto poly-L-lysine-coated cover slips ( $100 \mathrm{~g} / \mathrm{ml}$ poly-L-lysine for $2 \mathrm{~h}$ ). The plated neurons were maintained in HDMEM supplemented with $10 \%$ fetal calf serum for seven consecutive days at $37^{\circ} \mathrm{C}$ in an atmosphere of $5 \%$ $\mathrm{CO}_{2}-95 \%$ air. Cell morphology was monitored and described every day. After 7 days, cultures were washed in PBS, fixed in 4\% paraformaldehyde for $30 \mathrm{~min}$, and processed with antibodies against BrdU (1:150 dilution; Abcam, Cambridge, MA, USA) and $\beta$-III tubulin (1:500 dilution; Abcam). Briefly, cells were washed three times in PBS, permeabilized with $0.2 \%$ Triton X-100 in PBS for $10 \mathrm{~min}$ and incubated for $1 \mathrm{~h}$ at room temperature in a blocking solution of 5\% goat serum in PBS. The cells were then incubated overnight at room temperature in primary anitsera diluted in blocking solution. Subsequently, the cells were washed with PBS and incubated for $1 \mathrm{~h}$ in secondary antibodies (donkey anti-sheep Alexa 488, 1:400; Invitrogenand donkey anti-rabbit Cy3, 1:100; Jackson ImmunoResearch Laboratories). The cells were mounted in ProLong Gold (Invitrogen).

\section{FOOD INTAKE FOLLOWING CCK}

Rats were tested for their responsiveness to the satiety signal cholecystokinin octapeptide (CCK) at $30(n=4$ per group) and 300 days ( $n=12$ per group) post-treatment. All tests were conducted in the rats' home cages. Following an overnight fast ( $16 \mathrm{~h})$, rats were injected i.p. with CCK $(2 \mu \mathrm{g} / \mathrm{kg}$ in $0.9 \%$ sterile $\mathrm{NaCl}, \mathrm{PCK}-4100-\mathrm{v}$, Peptides International, Louisville, KY, USA).

A pre-weighed amount of chow was presented to each rat. At 15, 30 , and 60 min post-injection, the food was removed and weighed to determine the intake to the nearest $0.1 \mathrm{~g}$. Water was not withheld at any time during the experiment, and chow was returned to the cage following each test. To accustom rats to the procedure, the above protocol was repeated three times with sterile $\mathrm{NaCl}$ injections prior to any trial involving CCK injections. Additionally, any trial involving CCK was preceded and followed by a $\mathrm{NaCl}$ control trial.

\section{STATISTICAL ANALYSIS}

Data were analyzed by one-way repeated-measures analysis of variance (ANOVA) followed by the post hoc Bonferroni protected least significant difference (PLSD) using SigmaStat 3.5 software (Systat 
Software, Chicago, IL, USA). For the effect of CCK on food intake, CCK trials were compared to the previous $\mathrm{NaCl}$ control trial and tested for significance using a paired $t$-test. Significance was set at $p<0.05$. All values are expressed as means \pm standard error of the mean (SEM).

\section{RESULTS}

\section{NEURONAL REPLACEMENT IN ADULT NG FOLLOWS CAPSAICIN- INDUCED NEURONAL DEATH}

Immunofluorescent staining with an antibody against $\beta$-III tubulin revealed that vehicle-treated rats had approximately 7000 neurons per NG. The total number of neurons in vehicle-treated rats did not differ at 3-, 11-, 30-, and 60-days post-injection (6914 \pm 409 , $7024 \pm 351,6698 \pm 361$, and $7108 \pm 200$, respectively; Figure 1). NG collected 180- and 300-days post-vehicle injection contained fewer $\beta$-III tubulin-positive neurons as compared to 11-days post-injection $(5889 \pm 425, p=0.037$ and $6288 \pm 620, p=0.152$, respectively; Figure 1). NG from capsaicin-treated rats collected 3-, 11-, and 30-days post-injection contained significantly fewer $\beta$-III tubulin-positive neurons than time-matched vehicle-treated control rats. The lowest number of neurons occurred at 30-days post-capsaicin ( $3715 \pm 464, p=0.001$; Figure 1). However, by 60 -days post-injection, there was no difference in NG neuronal numbers between capsaicin- and vehicle-treated rats $(6956 \pm 149$ vs. $7108 \pm 200$; Figure 1). By 180-days post-capsaicin, the number of $\beta$-III tubulin-positive neurons was significantly greater than the number observed in control rats $(8067 \pm 664$ vs. $5889 \pm 425$, $p=0.002$; Figure 1). By 300-days post-injection, there was again no difference between capsaicin- and vehicle-treated rats (6444 \pm 797 vs. $6288 \pm 620$; Figure 1).

\section{CELLS WITHIN THE ADULT NG EXPRESS THE PROGENITOR CELL MARKER, NESTIN}

To determine if the NG contain progenitor cells capable of replacing neurons following capsaicin-induced death, we stained for the progenitor cell marker, nestin. Two distinct types of nestin-positive

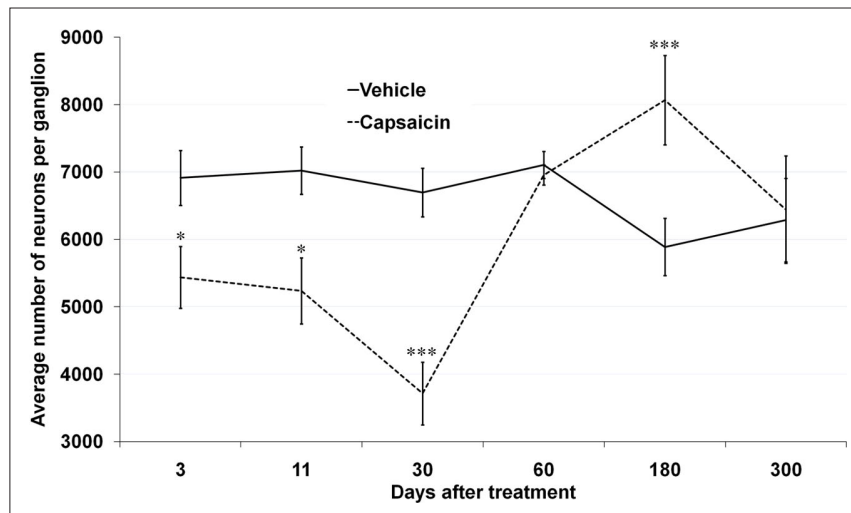

FIGURE 1 | Neuronal replacement in adult NG follows capsaicin-induced neuronal death. Total numbers of $\beta$-III tubulin stained neuronal profiles in left and right NG from vehicle- and capsaicin-treated rats. Note a decline and recovery in the total number of NG neurons over time followed by "overproduction" and decline to a control level after 300 days. ${ }^{*} p<0.05$, *** $p<0.001$ vs. Capsaicin. $n=4$ per group. cells were present in both vehicle- (Figure 2A) and capsaicin-treated (Figures 2B-D) rats. A large population of nestin-positive cells had glia-like profiles (Figure 2A, arrowheads). These cells were enveloping neuronal cell bodies and had flat processes that lay close to the neuronal plasma membrane, features indicative of satellite cells. An additional, much smaller population of nestinpositive cells (only a few per NG) had neuron-like profiles (Figures 2A,B,D, arrow), some with distinct neurite processes (Figures 2B,D, arrow). Double-labeling revealed that these nestin-positive neuron-like cells (Figures 2B,D, arrow) did not express $\beta$-III tubulin (Figure 2C, arrow).

\section{INCREASED CELL PROLIFERATION WITHIN ADULT NG FOLLOWS CAPSAICIN TREATMENT}

To determine if capsaicin treatment increases cell proliferation within the $\mathrm{NG}$, we investigated BrdU-incorporation and Ki-67-immunoreactivity. Immunofluorescent staining revealed BrdU-labeled cells in NG collected from both vehicle- and capsaicin-treated rats. However, capsaicin treatment significantly increased BrdU-incorporation in the nuclei of multiple cell types within NG collected 30- and 60-days post-injection. A significant portion these of BrdU-labeled nuclei were also positive for Ki-67 (Figure 3; arrows). The majority of these dual-labeled nuclei were located in satellite cells (Figure 3C). However, a few dual-labeled nuclei were located in neuron-like profiles (Figure 3C, arrow). The greatest difference in BrdU (Figure 3D; $8838 \pm 72$ vs. $13026 \pm 183$, $p<0.05$ ), Ki-67 (Figure 3E; $2694 \pm 68$ vs. $4320 \pm 105, p<0.05$ ),
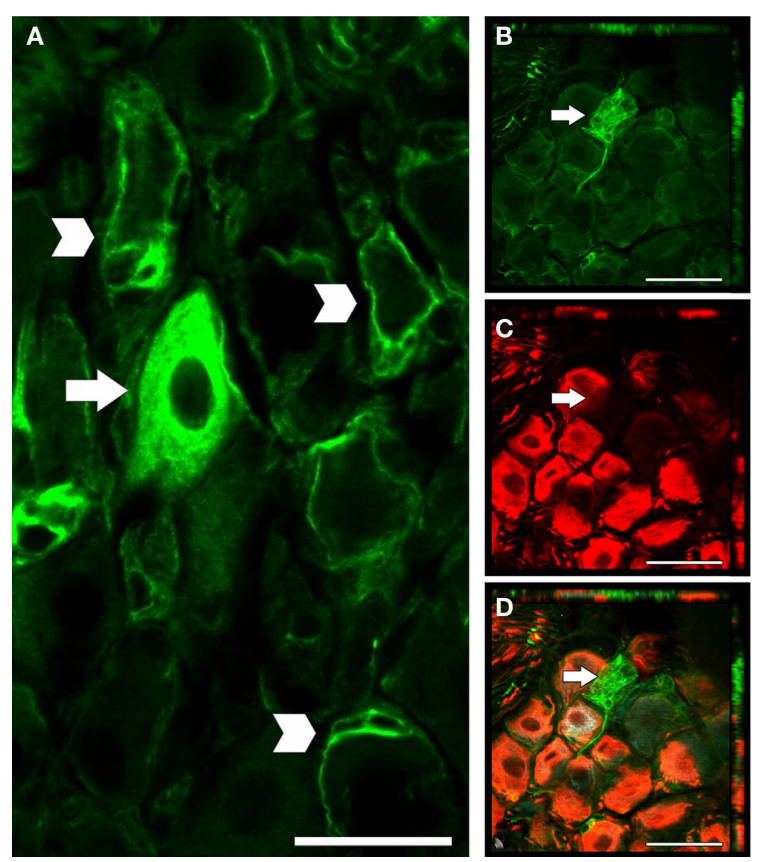

FIGURE 2 | Cells within the adult NG express the progenitor cell marker, nestin. Nestin (A, B, D) and $\beta$-III tubulin (C, D) immunoreactive cells in NG collected after vehicle treatment (A) and 300 days after capsaicin treatment (B-D). The nestin staining was present in neuron-like (arrows) and glia profiles (arrowheads). Nestin-immunoreactive neuron-like cells did not express $\beta$-III tubulin. $n=4$ per group. Scale bar $=20 \mu \mathrm{m}$. 


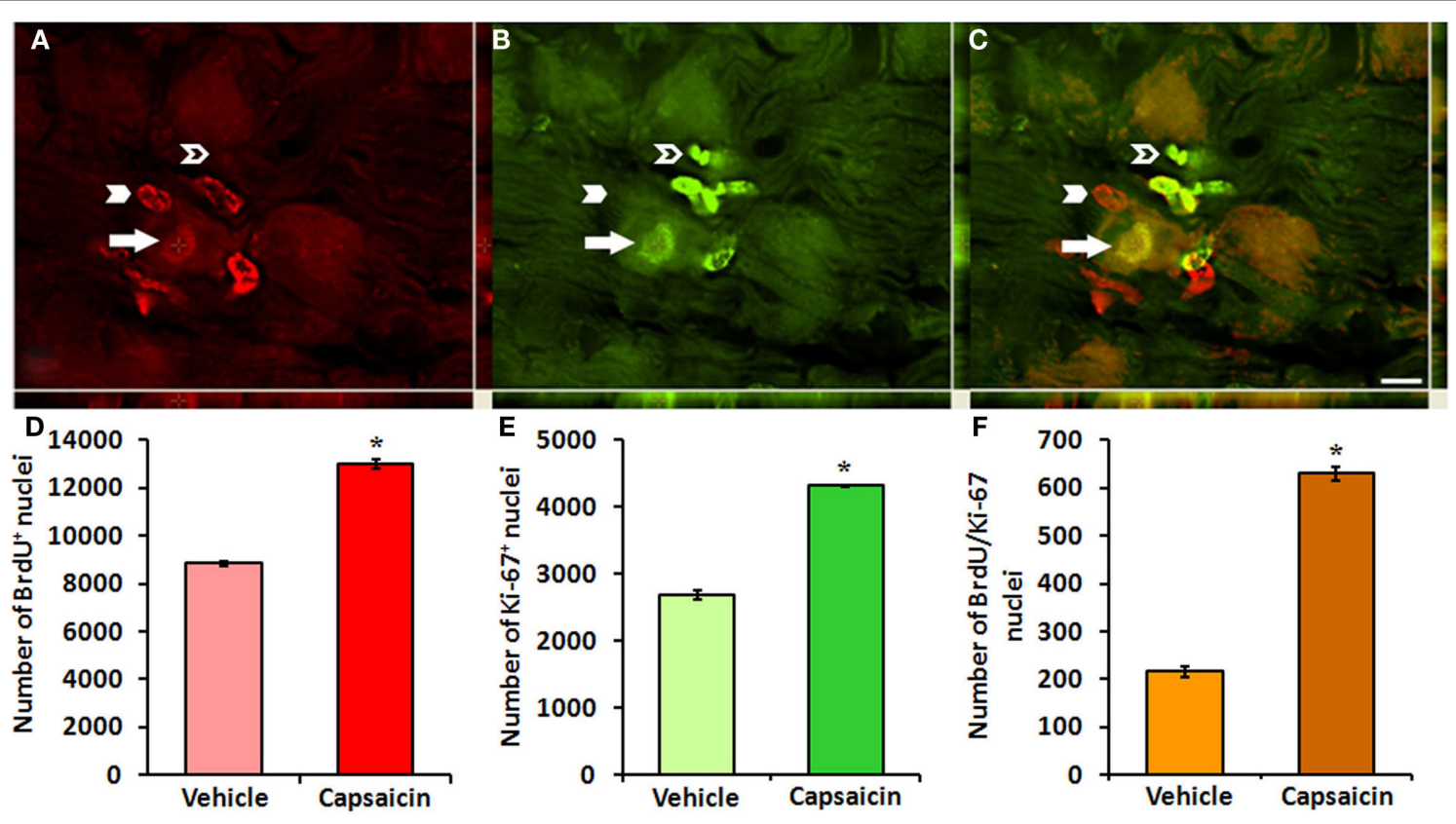

FIGURE 3 | Increased cell proliferation within adult NG follows capsaicin treatment. NG collected 30 days after capsaicin treatment revealed BrdU-(A) and Ki-67-labeled nuclei (B). Dual-labeled BrdU/Ki-67 nuclei belonged mostly to satellite cells. Very few neuronal-like profiles revealed BrdU/Ki-67-immunoreactive nuclei (C, arrow). A significant population of BrdU

and BrdU + /Ki-67 + dual-labeled cells between capsaicin- and vehicle-treated rats occurred 30-days post-injection (Figure 3F; $630 \pm 62$ vs. $216 \pm 94, p<0.05)$.

\section{DEVELOPMENTAL NEURONAL MORPHOLOGIES WERE FOUND IN CULTURES OF ADULT NG, COLLECTED FROM CAPSAICIN-TREATED RATS}

To monitor the extension of neurites from cells formed following capsaicin treatment, we cultured NG cells at multiple times post-injection. NG neurons in culture developed either singly (13-16 $\mu \mathrm{m}$ in diameter) or in small clumps $(9-11 \mu \mathrm{m}$ in diameter). A majority of the neuronal cell bodies remained spherical or ovoid, while others flattened onto satellite cells. Throughout the 7 days in culture, neuronal processes formed a network of increasing density. A majority of the neurons collected from vehicle-treated rats exhibited multipolar morphologies (Figure 4A). However, a significant portion of the neurons collected from capsaicin-treated rats revealed an early developmental spindleshaped bipolar morphology (Figure 4B). Transitional bell-shaped bipolar neurons were also present following capsaicin treatment (Figures 4C-E). Rarely, tripolar neurons were present as well following capsaicin treatment (Figure 4F). Both bipolar (Figures 4B-E) and tripolar (Figure 4F) neurons expressed the neuronal marker $\beta$-III tubulin, and most of them incorporated BrdU. $\beta$-III tubulin-positive neurons with bipolar or tripolar morphologies and BrdU-incorporation were also found in frozen sections of NG collected from capsaicin-treated rats (Figure 4G). Quantification analysis showed that capsaicin treatment significantly increased the number of bipolar morphology cells at 3 and 30 days (Figure 4H; $p<0.05$ ). single labeled nuclei (C, arrowhead) was found after capsaicin treatment. There were also few Ki-67 single labeled nuclei (C, hollow arrowhead). Capsaicin treatment significantly increased the numbers of BrdU (D), Ki-67 (E), and BrdU/Ki-67 colocalized nuclei (F). ${ }^{*} p<0.05$ vs. vehicle. $n=4$ per group. Scale bar $=10 \mu \mathrm{m}$.

\section{RESTORATION OF NMDA RECEPTOR EXPRESSION FOLLOWS CAPSAICIN- INDUCED DAMAGE OF NG NEURONS}

To determine if cells formed following capsaicin treatment exhibit pre-injury phenotypes necessary for normal signaling, we stained for the obligatory subunit of the NMDAR, NR1. NR1 was expressed within the cell body of neurons of all sizes throughout the NG. NR1-positive neurons were not relegated to any particular portion of the NG (Figures 5A-D). The number of NR1-positive neurons in vehicle-treated rats did not differ at 3-, 30-, 60-, 180-, and 300-days post-injection $(4821 \pm 128,4692 \pm 138,5015 \pm 68,4829 \pm 312$, and $5050 \pm 139$, respectively). Capsaicin treatment gradually reduced the number of NR1-positive neurons to a minimum of $1296 \pm 111$ at 180 -days post-injection (Figure $5 \mathrm{E} ; p<0.01$ at day $3, p<0.001$ at days 30,60 , and 180 ). However, by 300 -days post-capsaicin, the number of NR1-positive neurons was not statistically different than that found in the vehicle-treated controls ( $4776 \pm 352$ vs. $5050 \pm 139$; Figures 5C-E). The message for NR1 followed a similar pattern of disruption and subsequent restoration. Three days post-capsaicin, NR1 mRNA was reduced to $66.5 \pm 4.5 \%$ of time-matched vehicletreated controls (Figure 5F, $p=0.047$ ). Thirty days post-capsaicin, the average level of NR1 mRNA was still reduced $(70.0 \pm 8.9 \%$, $p=0.081)$. However, by 60 -days post-capsaicin, NR1 mRNA had risen to the level of vehicle-treated controls $(95.1 \pm 13.9 \%)$.

\section{RESTORATION IN THE NUMBER OF CENTRAL PROJECTIONS FOLLOWS CAPSAICIN-INDUCED DAMAGE TO NG NEURONS}

To determine if the number of central projections is restored following capsaicin-induced reduction, we stained for the synaptic marker, synaptophysin, within the NTS. Quantitative stereological 

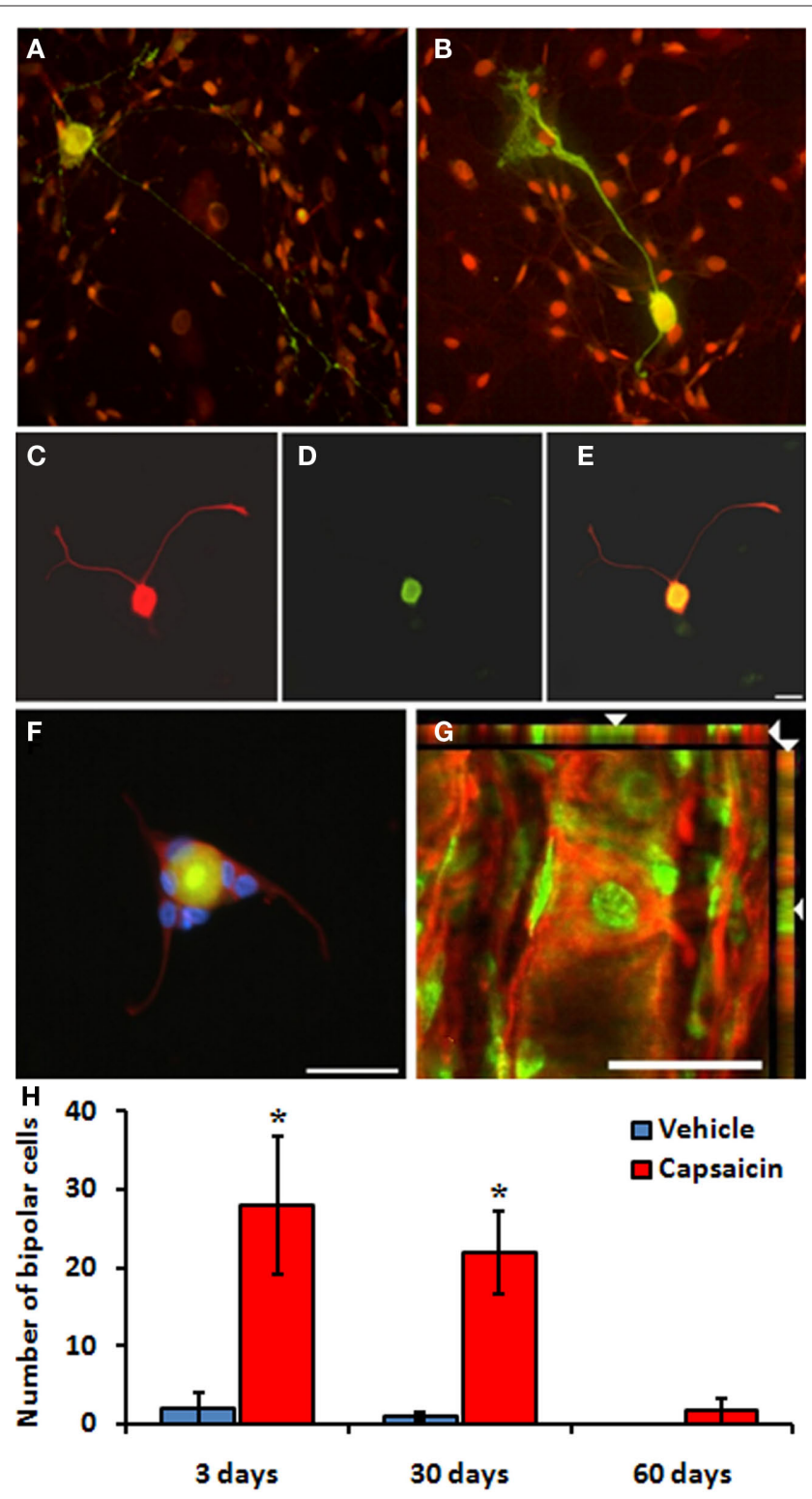

days

30 days

FIGURE 4 | Developmental neuronal morphologies were found in cultures of adult NG collected from capsaicin-treated rats. (B-F)

Representative images of cultured NG neuronal morphology following vehicle (A) and capsaicin (B) treatment. (A) Example of a typical neuron cultured from a vehicle-treated NG exhibiting multipolar morphology. (B) Example of developmental spindle-shaped bipolar morphology expressing $\beta$-III tubulin (green; red = DAPI). (C-E) Example of transitional bell-shaped morphology. These neurons also expressed $\beta$-III tubulin (C) and incorporated BrdU (D; $\mathrm{E}=$ merged). (F) Example of a tripolar neuron surrounded by DAPI-labeled glial nuclei (red $=\beta$-III tubulin; green = BrdU; blue = DAPI). (G) Example of a mature neuron expressing $\beta$-III tubulin (red) and incorporating BrdU (green) within a frozen section of the NG following capsaicin treatment. The numbers of bipolar cells were significantly increased at day 3 and day 30 after capsaicin treatment $\mathbf{( H )} .{ }^{*} p<0.05$ vs. vehicle. $n=3$ per group. Scale bar $=20 \mu \mathrm{m}$.

analysis revealed a slightly uneven distribution of synaptophysinpositive synapses within the NTS. Specifically, the gelatinous and the medial regions of the NTS were more densely populated as
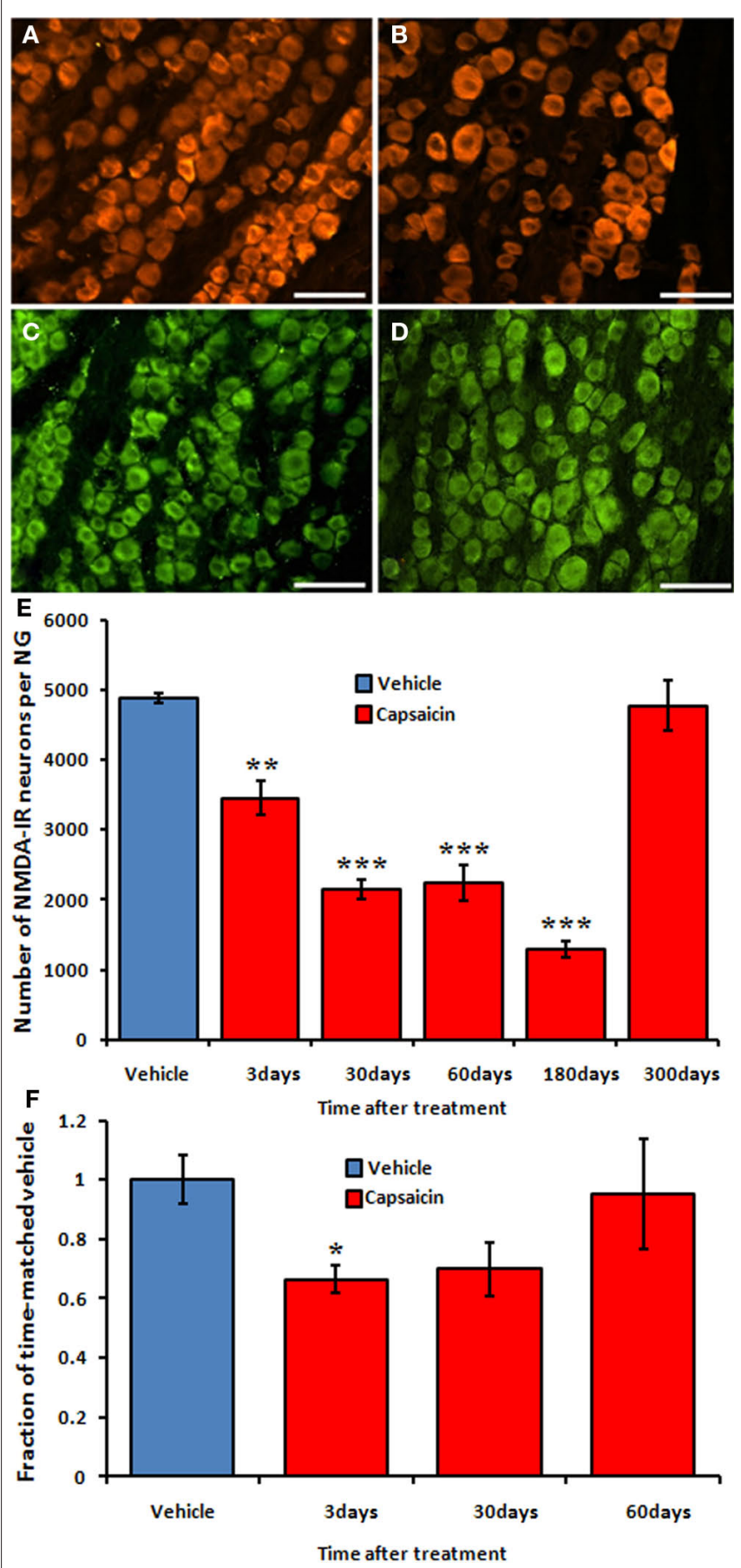

FIGURE 5 | (A,B) NR1-IR neurons in NG collected 180 days after vehicle (A) and capsaicin (B) treatment. (C,D) NR1-IR neurons in NG collected 300 days after vehicle (C) and capsaicin (D) treatment. A decline of NR1 subunit of NMDA receptor was observed up to 180 days after capsaicin treatment (red bars, E) when compared to vehicle-treated rats (blue bar, E). NR1 subunit reactivity was restored to control levels at 300 days after capsaicin treatment (red bar, E). (F) Decrease and restoration of the relative expression of NR1 mRNA using RT-PCR. Blue bar represents pooled data from vehicle-treated rats; red bars represent data from capsaicin-treated rats at 3-, 30-, and 60-days post-injection. ${ }^{*} p<0.05,{ }^{*} p<0.01,{ }^{* *} p<0.001$ vs. vehicle. $n=3-4$ per group. Scale bar $=50 \mu \mathrm{m}$. 
compared to the lateral NTS (Figures 6A-D). The number of synaptophysin-positive synapses within the NTS of vehicle-treated controls did not differ at 3-, 30-, 60-, and 300-days post-injection $(4.56 \pm 0.36,4.58 \pm 0.44,3.78 \pm 0.33$, and $4.46 \pm 0.17$ million, respectively; Figure 6E). Capsaicin treatment significantly decreased synaptophysin-positive synapses 3-days post-injection $(1.52 \pm 0.41$ million, $p=0.016)$. This decrease remained significantly lower 30 -days post-capsaicin $(2.46 \pm 0.40$ million, $p<0.05)$ and 60 -days post-capsaicin $(2.39 \pm 0.39$ million, $p<0.05)$. However, by 300 -days post-injection, the number of synaptophysin-

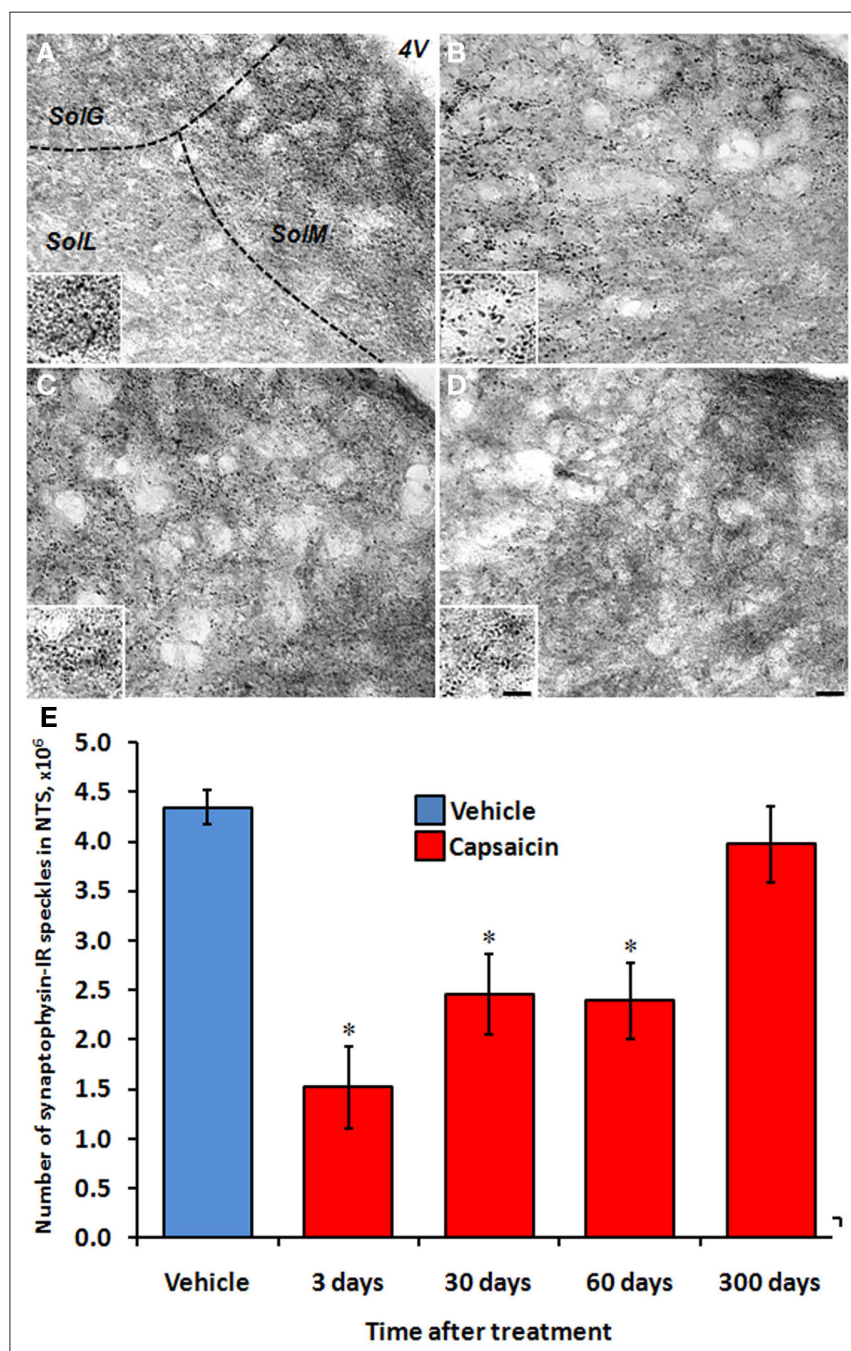

FIGURE 6 | Restoration in the number of central projections follows capsaicin-induced damage to NG neurons. (A-D) Representative images of the same anatomical level of the NTS (bregma-13.3 mm) 3-days post-vehicle (A), 3-days post-capsaicin (B), 300-days post-vehicle (C), and 300-days post-capsaicin (D) showing the decrease and restoration in synaptophysin immunoreactivity following capsaicin. Inset shows the same representative NTS at higher magnification. (E) Quantification of synaptophysin-positive synapses in the NTS using unbiased stereology. Blue bar represents pooled vehicle-treated rats; red bars represent capsaicin-treated rats at 3-, 30-, 60-, and 300-days post-injection. ${ }^{*} p<0.05$ vs. vehicle. $n=4$ per group. $4 \mathrm{~V}$, 4 th ventricle; SolG, gelatinous NTS; SolM, medial NTS; SolL, lateral NTS. Scale bars $=20 \mu \mathrm{m}$ (large image) and $8 \mu \mathrm{m}$ (inset). positive synapses within the NTS of capsaicin-treated rats did not significantly differ from time-matched vehicle-treated controls (3.97 \pm 0.38 million).

\section{RESTORATION OF VAGAL AFFERENT SIGNALING FOLLOWS CAPSAICIN- INDUCED DISRUPTION}

To determine if restored neuronal numbers, phenotypes, and projections correlate with restored function, we monitored food intake following administration of the satiety-inducing hormone, CCK. Vehicle-treated rats decreased their food intake by approximately $2.5 \mathrm{~g}$ over $1 \mathrm{~h}$ when injected with CCK. In contrast, when food intake was measured 30-days post-capsaicin, rats did not decrease their food intake in response to CCK (Figure 7A). However, by 300-days post-injection, $50 \%$ of capsaicin-treated rats ( 6 of 12) significantly decreased their food intake in response to CCK by $0.9 \mathrm{~g}$ at both 15 and $30 \mathrm{~min}$ ( $p=0.002$ and 0.033 , respectively; Figure 7B).

\section{DISCUSSION}

The present study shows that systemic capsaicin treatment significantly decreased the number of $\beta$-III tubulin-positive neurons within the NG. However, by 60 -days post-capsaicin, the total number of neurons within the NG was not different from vehicletreated controls. This suggests that new neurons were added to

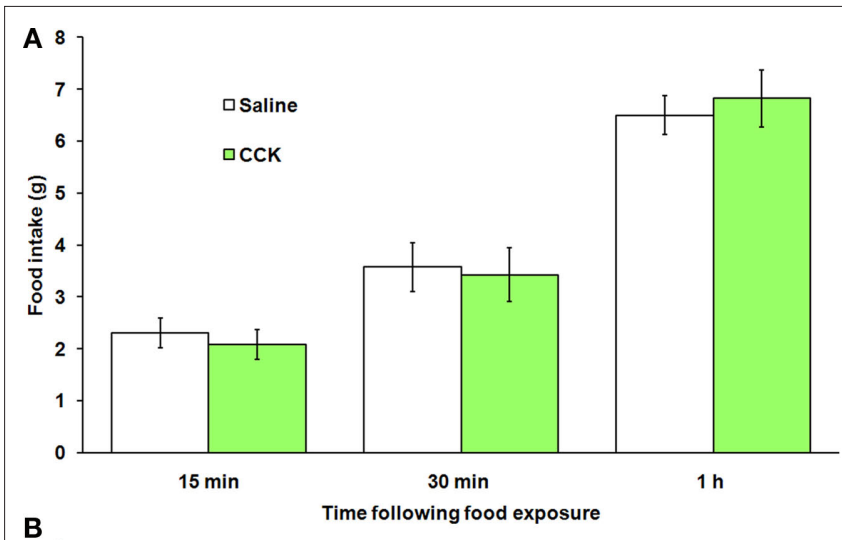

B

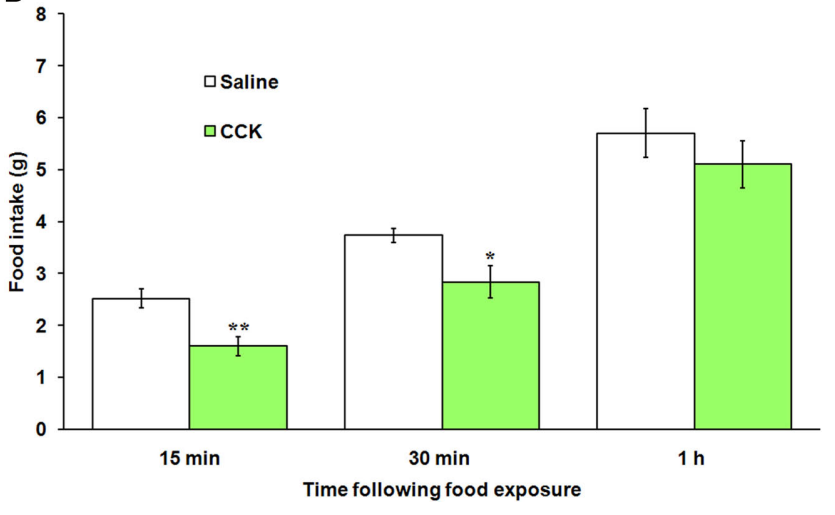

FIGURE 7 | Restoration of vagal afferent signaling follows capsaicininduced disruption. (A) Food intake of 30-days post-capsaicin rats after a control injection of $0.9 \% \mathrm{NaCl}$ (white bars) or $2 \mu \mathrm{g} / \mathrm{kg}$ CCK (green bars) showing no response to CCK in all rats tested. (B) At 300-days post-capsaicin, 6 out of 12 rats significantly decreased food intake at 15 and 30 min after CCK injection. ${ }^{*} p<0.05,{ }^{*} p<0.01$ vs. saline. $n=4$ at day $30, n=12$ at day 300 . 
the NG following capsaicin-induced neuronal death. By 180-days post-capsaicin, the number of $\beta$-III tubulin-positive neurons was significantly greater than the number in control rats. By 300-days post-injection, there was no difference between groups. This overproduction of neurons between 60 and 180-days post-capsaicin followed by the decrease to the control number by 300 days resembles the changes in neuronal numbers observed during the perinatal period of PNS development (Oppenheim, 1991). This finding is in agreement with our previously published results showing that the total number of DAPI-labeled neuronal-like nuclei in NG from capsaicin-treated rats was not different from controls by 60 -days post-injection (Czaja et al., 2008). Moreover, our results revealed age-related decrease in the number of neurons in NG collected from vehicle-treated controls. The effect of age on neuronal numbers in sensory ganglia have been evaluated in a number of experiments, however, the work has failed to bring conclusive data to reveal an age-dependent change. The recently published studies of vagal and spinal afferents innervating gastrointestinal tract (Phillips et al., 2010) revealed dystrophic and regressive changes with aging, what may support our observations. Given these findings, we conclude that capsaicin-induced neuronal loss is followed by a large scale neuronal replacement in the adult NG. In the CNS several studies support the hypothesis of insult-induced neurogenesis and show that damage to the adult nervous system induces factors and mechanisms that control neuronal proliferation, migration, differentiation, and connectivity during development (Ramaswamy et al., 2005; Zhu et al., 2005; Tan et al., 2010). However, an induction of adult neurogenesis that results in addition or replacement of a large proportion of these neurons has not been reported in adult mammalian brain, although it does occur in some non-mammalian vertebrates (Chapouton et al., 2007).

To account for the source of the neuronal replacement following capsaicin-induced death, we looked for the presence of the stem cell marker, nestin. Here we show that nestin-positive cells are present in NG collected from both the vehicle- and capsaicin-treated rats. A small number of these nestin-positive cells revealed neuronal profiles, however, they did not express $\beta$-III tubulin. Such cells expressing markers of neuronal progenitors and not recognized as mature neurons were previously reported in the trigeminal and DRG (Farel, 2003; Lagares et al., 2007; Li et al., 2007; Singh et al., 2009). The presence of these cells suggests that delayed maturation may be partially responsible for the neuronal replacement we observed in adult rat NG. It is also possible that after injury NG cells re-express nestin and recapitulate early development signaling that was previously reported in the CNS (Kriegstein and Alvarez-Buylla, 2009; Berninger, 2010). However this fate switch of differentiated cells is less likely to occur in the adult NG because our results show that satellite glia made up the majority of nestin-positive cells in both the capsaicin-treated rats and vehicle-treated controls. However, further studies employing lineage-tracing methods to label the proliferating cells and to follow their development after damage are needed to rule out the possibility that after capsaicininduced death of TRPV1-expressing neurons, glia in NG undergo lineage reprogramming (Buffo et al., 2008) and differentiate into neurons. The presence of the large number of potential progenitor cells, as shown by the expression of nestin, suggests that the adult NG has a robust neurogenic potential.
To further examine the neurogenic potential of the adult NG, we quantified both BrdU-incorporation and the mitotic marker, Ki-67. Capsaicin treatment significantly increased BrdU-incorporation within the nuclei of multiple cell types in NG collected 30- and 60 -days post-injection. Importantly, a significant portion of BrdUlabeled nuclei were simultaneously immunoreactive for Ki-67. We previously reported that two months following capsaicin-induced neuronal death, BrdU was detected in more than $10 \%$ of neuronal nuclei, indicating that cells within the NG had reentered the $S$ phase of the cell cycle and differentiated into mature neurons (Czaja et al., 2008). In the present study, dual-labeled BrdU/Ki-67 profiles represent a population of proliferating NG cells in an active cell cycle, because BrdU is incorporated during DNA replication (Nowakowski et al., 1989; Wojtowicz and Kee, 2006), and Ki-67 is a nuclear antigen expressed only during mitosis (Kee et al., 2002). The vast majority of these dual-labeled nuclei belonged to satellite glia cells. It has been previously reported that axonal damage without neuronal loss induces satellite cell proliferation in sensory ganglia (Lu and Richardson, 1991; Wen et al., 1994). In our model, we observed a significant neuronal loss followed by neuronal replacement and restoration of neuronal numbers. Therefore, we conclude that systemic capsaicin treatment during adulthood stimulates satellite cells to undergo symmetric or asymmetric cell division and differentiate into new satellite cells and sensory neurons.

To monitor the development and neurite outgrowth of these new sensory neurons over time, we cultured NG neurons following capsaicin treatment. We found that capsaicin treatment significantly increased the number of neurons with bipolar or tripolar morphology. Among the bipolar neurons, transitional "bell-shaped" bipolar neurons were detected. These observations are significant because sensory ganglion neurons undergo a unique transformation from bipolar to pseudounipolar cells during development (Matsuda et al., 1998). Moreover, both the bipolar and tripolar cells expressed $\beta$-III tubulin, confirming their neuronal nature, and most of them incorporated BrdU, showing that they contain newly replicated DNA. Due to these findings, we conclude that capsaicin treatment induces the progenitor cells present in adult NG to proliferate and proceed through developmental stages normally observed only during the perinatal period.

To determine if newly formed neurons express pre-injury phenotypes, we quantified the expression of the obligatory subunit of the NMDAR, NR1. Our previously published data revealed that the NR1 subunit was present in almost all NG neurons (Czaja et al., 2006a). Here, we show that capsaicin treatment gradually reduces the number of NR1-positive neurons out to 180-days post-injection. However, by 300-days after capsaicin treatment, the number of NR1-positive neurons was not different than the vehicle-treated controls. The initial decrease in NR1 immunoreactivity can be explained by the fact that capsaicin is killing approximately $50 \%$ of NG neurons within the first 30 days. However, low numbers of NR1-positive neurons were observed up to 180-days post-capsaicin, the total number of NG neurons was restored by 60 -days postinjection. Interestingly, the mRNA message for NR1 followed a similar pattern of decrease and subsequent restoration, but was restored to control levels by 60 -days post-capsaicin. Together, these data show that new NG neurons initially transcribe the message for NR1, but any translated protein is transported away from the cell 
body toward the extending peripheral and central processes. This conclusion is consistent with the NMDAR's established role during key steps of neural development and synaptogenesis (Contestabile, 2000) and supported by the correlation with the restoration of central connections within the NTS discussed below.

Because neurons generated following capsaicin treatment must incorporate into meaningful neural circuitry to survive and function, we monitored the number of synapses within the primary target of NG vagal afferents, the NTS. Capsaicin treatment significantly decreased NTS-synaptophysin immunoreactivity up to 60 -days post-injection. This observed decrease in the number of synapses within the NTS following capsaicin treatment is most likely the effect of two main mechanisms: (1) damaged but surviving NG neurons withdraw their central terminals from the NTS and (2) as systemic capsaicin kills approximately $50 \%$ of NG neurons (Czaja et al., 2008), their central terminals within the NTS are degraded. Decreases in the number of central synapses following peripheral axotomy have been previously described in the spinal innervation (Brannstrom and Kellerth, 1999; White and Kocsis, 2002; Sun et al., 2006). Like in the vagal innervation, losses of the central synaptic connections were eventually restored when the reinnervation of the peripheral target organs was completed (Brannstrom and Kellerth, 1999). By 300-days post-injection, the number of synaptophysin-positive synapses within the NTS of capsaicin-treated rats did not significantly differ from controls, suggesting that central vagal projections were restored. This restoration in central projections may be due to one or more of three mechanisms: (1) regeneration of the withdrawn central terminals of injured but surviving NG neurons, (2) the sprouting of regenerating or uninjured central axons, or (3) the birth of new neurons, followed by the successful extension of central projections into the NTS. Because peripheral projections are also restored following capsaicin-induced damage to both the spinal (Gallaher et al.,

\section{REFERENCES}

Arora, D. K., Cosgrave, A. S., Howard, M. R., Bubb, V., Quinn, J. P., and Thippeswamy, T. (2007). Evidence of postnatal neurogenesis in dorsal root ganglion: role of nitric oxide and neuronal restrictive silencer transcription factor. J. Mol. Neurosci. 32, 97-107.

Berninger, B. (2010). Making neurons from mature glia: a far-fetched dream? Neuropharmacology 58, 894-902.

Brannstrom, T., and Kellerth, J. O. (1999). Recovery of synapses in axotomized adult cat spinal motoneurons after reinnervation into muscle. Exp. Brain Res. 125, 19-27.

Buffo, A., Rite, I., Tripathi, P., Lepier, A., Colak, D., Horn, A. P., Mori, T., and Gotz, M. (2008). Origin and progeny of reactive gliosis: a source of multipotent cells in the injured brain. Proc. Natl. Acad. Sci. U.S.A. 105, 3581-3586.

Calhoun, M. E., Jucker, M., Martin, L. J., Thinakaran, G., Price, D. L., and Mouton, P. R. (1996). Comparative evaluation of synaptophysin-based

2010) and vagal (Ryu et al., 2010) viscerosensory innervation, it is possible that newly formed neurons make connections that would enable them to be functional.

To determine if restored central projections do correlate with restored function, we tested the response to the satiety hormone, CCK. Capsaicin has been previously shown to abolish the satietyinducing effect of CCK in rats (South and Ritter, 1988; Ritter et al., 1989). Our test was consistent with these reports in that 30-days post-capsaicin rats did not decrease their food intake in response to CCK. However, by 300 -days post-injection, $50 \%$ of capsaicin-treated rats significantly decreased their food intake in response to CCK, indicating that the satiety-inducing effect of CCK was restored in these rats. The recovery of compromised satiety signaling correlates with both the restoration of synaptic connections within NTS as well as NR1 immunoreactivity within the NG.

The results of this study show that the PNS harbors progenitor cells that are capable of proliferating and differentiating into new neurons. Subsequently, these newly formed neurons are capable of integrating into the PNS circuitry and contributing to the restoration of lost functions. Future work will elucidate the mechanisms responsible for the induction of proliferation and differentiation of these progenitor cells. It is our hope that this knowledge may be used to treat cases of damage and degenerative disease within the nervous system.

\section{ACKNOWLEDGMENTS}

This research was supported by WSU Startup Funds and George W. Bagby Research Fund. We would like to thank the Seattle Chapter of the ARCS Foundation, Inc. and the Aven Foundation for their graduate student support. Authors would like to thank Dr. S. Simasko, Dr. H. Zhao, and D. Kinch for their expertise regarding cell culture experiments.

Czaja, K., Burns, G. A., and Ritter, R. C. (2008). Capsaicin-induced neuronal death and proliferation of the primary sensory neurons located in the nodose ganglia of adult rats. Neuroscience 154, 621-630.

Czaja, K., Ritter, R. C., and Burns, G. A. (2006a).N-methyl-D-aspartate receptor subunit phenotypes of vagal afferent neurons in nodose ganglia of the rat. J. Comp. Neurol. 496, 877-885.

Czaja, K., Ritter, R. C., and Burns, G. A. (2006b). Vagal afferent neurons projecting to the stomach and small intestine exhibit multiple N-methylD-aspartate receptor subunit phenotypes. Brain Res. 1119, 86-93.

Dockray, G. J. (2009). Cholecystokinin and gut-brain signalling. Regul. Pept. 155, 6-10

Farel, P. B. (2003). Late differentiation contributes to the apparent increase in sensory neuron number in juvenile rat. Brain Res. Dev. Brain Res. 144, 91-98.

Gallaher, Z. R., Larios, R. M., Ryu, V., Sprunger, L. K., and Czaja, K. (2010). Recovery of viscerosensory innervation from the dorsal root ganglia of the adult rat following capsaicin-induced injury. J. Comp. Neurol. 518, 3529-3540.

Geuna, S., Borrione, P., and Filogamo, G. (2002). Postnatal histogenesis in the peripheral nervous system. Int. J. Dev. Neurosci. 20, 475-479.

Gillespie, B. R., Burns, G. A., and Ritter, R. C. (2005). NMDA Channels control meal size via central vagal afferent terminals. Am. J. Physiol. Regul. Integr. Comp. Physiol. 289, R1504-R1511.

Hiura, A. (2000). Neuroanatomical effects of capsaicin on the primary afferent neurons. Arch. Histol. Cytol. 63, 199-215.

Holzer, P. (1991). Capsaicin: cellular targets, mechanisms of action, and selectivity for thin sensory neurons. Pharmacol. Rev. 43, 143-201.

Jin, Y. H., Cahill, E. A., Fernandes, L. G., Wang, X., Chen, W., Smith, S. M., and Andresen, M.C. (2010). Optical tracking of phenotypically diverse individual synapses on solitary tract nucleus neurons. Brain Res. 1312, 54-66. 
Kee, N., Sivalingam, S., Boonstra, R., and Wojtowicz, J. M. (2002). The utility of Ki-67 and BrdU as proliferative markers of adult neurogenesis. J. Neurosci. Methods 115, 97-105.

Kempermann, G., Jessberger, S., Steiner, B., and Kronenberg, G. (2004). Milestones of neuronal development in the adult hippocampus. Trends Neurosci. 27, 447-452.

Kriegstein, A., and Alvarez-Buylla, A. (2009). The glial nature of embryonic and adult neural stem cells. Annu. Rev. Neurosci. 32, 149-184.

Lagares, A., Li, H. Y., Zhou, X. F., and Avendano, C. (2007). Primary sensory neuron addition in the adult rat trigeminal ganglion: evidence for neural crest glio-neuronal precursor maturation. J. Neurosci. 27, 7939-7953.

Li, H. Y., Say, E. H., and Zhou, X. F. (2007). Isolation and characterization of neural crest progenitors from adult dorsal root ganglia. Stem Cells 25, 2053-2065.

Liu, M. T., Kuan, Y. H., Wang, J., Hen, R., and Gershon, M. D. (2009). 5-HT4 receptor-mediated neuroprotection and neurogenesis in the enteric nervous system of adult mice. J. Neurosci. 29, 9683-9699.

Lu, X., and Richardson, P. M. (1991). Inflammation near the nerve cell body enhances axonal regeneration. J. Neurosci. 11, 972-978.

Matsuda, S., Kobayashi, N., Mominoki, K., Wakisaka, H., Mori, M., and Murakami, S. (1998). Morphological transformation of sensory ganglion neurons and satellite cells. Kaibogaku. Zasshi. 73, 603-613.

Milner, T. A., and Pickel, V. M. (2003). Receptor targeting in medullary nuclei mediating baroreceptor reflexes1. Cell. Mol. Neurobiol. 23, 751-760.

Ming, G. L., and Song, H. (2005). Adult neurogenesis in the mammalian central nervous system. Annu. Rev. Neurosci. 28, 223-250.

Mohammed, H. A., and Santer, R. M. (2001). Total neuronal numbers of rat lumbosacral primary afferent neurons do not change with age. Neurosci. Lett. 304, 149-152.

Namaka, M.P., Sawchuk, M., MacDonald, S. C., Jordan, L. M., and Hochman, S. (2001). Neurogenesis in postnatal mouse dorsal root ganglia. Exp. Neurol. 172, 60-69.

Nowakowski, R. S., Lewin, S. B., and Miller, M. W. (1989). Bromodeoxyuridine immunohistochemical determination of the lengths of the cell cycle and the DNA-synthetic phase for an anatomically defined population. J. Neurocytol. 18, 311-318.

Oppenheim, R. W. (1991). Cell death during development of the nervous system. Annu. Rev. Neurosci. 14, 453-501.

Peters, J. H., Ritter, R. C., and Simasko, S. M. (2006). Leptin and CCK selectively activate vagal afferent neurons innervating the stomach and duodenum. Am. J. Physiol. Regul. Integr. Comp. Physiol. 290, R1544-R1549.

Petrus, D. S., Fabel, K., Kronenberg, G., Winter,C.,Steiner,B., and Kempermann, G. (2009). NMDA and benzodiazepine receptors have synergistic and antagonistic effects on precursor cells in adult hippocampal neurogenesis. Eur. J. Neurosci. 29, 244-252.

Phillips, R. J., Walter, G. C., and Powley, T. L. (2010). Age-related changes in vagal afferents innervating the gastrointestinal tract. Auton. Neurosci. 153, 90-98.

Popken, G. J., and Farel, P. B. (1997). Sensory neuron number in neonatal and adult rats estimated by means of stereologic and profile-based methods. J. Comp. Neurol. 386, 8-15.
Ramaswamy, S., Goings, G.E., Soderstrom, K. E., Szele, F. G., and Kozlowski, D A. (2005). Cellular proliferation and migration following a controlled cortical impact in the mouse. Brain Res. 1053, 38-53.

Ritter, R. C., Ritter, S., Ewart, W. R., and Wingate, D. L. (1989). Capsaicin attenuates hindbrain neuron responses to circulating cholecystokinin. Am. J. Physiol. 257, R1162-R1168.

Ritter,S., and Dinh, T.T.(1988). Capsaicininduced neuronal degeneration: silver impregnation of cell bodies, axons, and terminals in the central nervous system of the adult rat. J. Comp. Neurol. 271, 79-90.

Ryu, V., Gallaher, Z., and Czaja, K. (2010). Plasticity of nodose ganglion neurons after capsaicin- and vagotomyinduced nerve damage in adult rats. Neuroscience 167, 1227-1238.

Singh, R. P., Cheng, Y. H., Nelson, P., and Zhou, F.C. (2009). Retentive multipotency of adult dorsal root ganglia stem cells. Cell Transplant. 18, 55-68.

South, E. H., and Ritter, R. C. (1988). Capsaicin application to central or peripheral vagal fibers attenuates CCK satiety. Peptides 9, 601-612.

Sun, T., Xiao, H. S., Zhou, P. B., Lu, Y. J., Bao, L., and Zhang, X. (2006). Differential expression of synaptoporin and synaptophysin in primary sensory neurons and up-regulation of synaptoporin after peripheral nerve injury. Neuroscience 141 , 1233-1245.

Tan, Y. F., Preston, E., and Wojtowicz, J. M. (2010). Enhanced post-ischemic neurogenesis in aging rats. Front. Neurosci. 4. doi: 10.3389/fnins.2010.00163

Wen, J. Y., Morshead, C. M., and van der Kooy, D. (1994). Satellite cell proliferation in the adult rat trigeminal ganglion results from the release of a mitogenic protein from explanted sensory neurons. J. Cell Biol. 124, 1005-1015.

White, F. A., and Kocsis, J. D. (2002). A-fiber sprouting in spinal cord dorsal horn is attenuated by proximal nerve stump encapsulation. Exp. Neurol. 177, 385-395.

Wojtowicz, J.M., and Kee, N. (2006). BrdU assay for neurogenesis in rodents. Nat. Protoc. 1, 1399-1405.

Zhu, L. L., Zhao, T., Li, H. S., Zhao, H., Wu, L. Y., Ding, A. S., Fan, W. H., and Fan, M. (2005). Neurogenesis in the adult rat brain after intermittent hypoxia. Brain Res. 1055, 1-6.

Conflict of Interest Statement: The authors declare that the research was conducted in the absence of any commercial or financial relationships that could be construed as a potential conflict of interest.

Received: 29 September 2010; accepted: 20 January 2011; published online: 02 February 2011.

Citation: Gallaher ZR, Ryu V, Larios RM, Sprunger LK and Czaja K (2011) Neural proliferation and restoration of neurochemical phenotypes and compromised functions following capsaicin-induced neuronal damage in the nodose ganglion of the adult rat. Front. Neurosci. 5:12. doi: 10.3389/fnins.2011.00012

This article was submitted to Frontiers in Neurogenesis, a specialty of Frontiers in Neuroscience.

Copyright (C) 2011 Gallaher, Ryu, Larios, Sprunger and Czaja. This is an open-access article subject to an exclusive license agreement between the authors and Frontiers Media SA, which permits unrestricted use, distribution, and reproduction in any medium, provided the original authors and source are credited. 